\title{
SYNTHESIS AND CHARACTERIZATION OF NOVEL ORGANOBISMUTH FOR ANTIMICROBIAL AND ANTITUMOR STUDIES
}

\author{
DIPTI MANI TRIPATHI, RAVI KANT, KRISHNA SRIVASTAVA* \\ Department of Chemistry, Faculty of Chemical Sciences, Shri Ramswaroop Memorial University, Lucknow, Uttar Pradesh, India. \\ Email: Krishna.cy@srmu.ac.in
}

Received: 23 September 2019, Revised and Accepted: 07 March 2020

\begin{abstract}
Objectives: The major objective of this manuscript is to present synthesis and biomedical screening of some organic derivatives of bismuth having general formula $\left(\mathrm{R}_{3} \mathrm{BiL}_{2}\right)$ by the method reported and characterized with the help of M.P., elemental, I.R., and NMR spectral analysis along with their
\end{abstract} antimicrobial and in vitro antitumor activity against human breast (MCF-7) and mammary cancer (EVSA-7) cell line.

Methods: All the newly organobismuth having general formula $\left[\mathrm{R}_{3} \mathrm{BiL}_{2}\right]$ were synthesised by the method reported especially using oxidative addition and complexation reactions.

Results: It was found that organobismuth compounds have trigonal bipyramidal structure as per their elemental and spectral analysis and show potentiality as antimicrobial and antitumor agents.

Conclusion: The newly synthesized organobismuth(V)substituted carboxylates were fully characterized chemically to ascertain their structure by sophisticated instrumental and spectral analysis resulted as trigonal bipyramidal structure. The compounds were also screened $1^{\text {st }}$ time for antitumor and antimicrobial studies. The observations clearly indicated that organobismuth carboxylates show potent antimicrobial and antitumor activity.

Keywords: Organobismuth, Antimicrobial, Antitumor.

(C) 2020 The Authors. Published by Innovare Academic Sciences Pvt Ltd. This is an open access article under the CC BY license (http://creativecommons. org/licenses/by/4. 0/) DOI: http://dx.doi.org/10.22159/ajpcr.2020.v13i5.35854

\section{INTRODUCTION}

It is well reported that bismuth compounds have attracted considerable interest due to their biological and medicinal utility [1-5]. They have been utilized from more than 2 centuries in the treatment of gastrointestinal disorders such as dyspepsia, diarrhea, and peptic ulcer [6-9]. Bismuth salts such as colloidal bismuth sub-citrate, bismuth sub-salicylate, and ranitidine bismuth citrate are common agents used for Helicobacter pylori eradication therapy and therefore promoted these compounds as antimicrobials $[10,11]$. The utility of bismuth formulations has motivated many studies into their possible mechanism of action and to the discovery of their biological targets. In search of antiproliferative studies, a variety of organobismuth compounds has been synthesized and tested in vitro for their antitumor activity along with their antimicrobial activity [12-15]. The present manuscript describes the synthesis, structural, antimicrobial, and antitumor studies of some novel fluorine-based organic derivatives of bismuth. The compounds were synthesized by the method reported earlier and characterized with the help of M.P., elemental, I.R., and NMR spectral analysis along with their antimicrobial studies, against different pathogenic bacterial and fungal strains and in vitro antitumor activity against human breast (MCF-7) and mammary cancer (EVSA-7) cell line and found that compounds have potentiality as antitumor and antimicrobial agents.

\section{METHODS}

All the newly organobismuth having general formula $\left[\mathrm{R}_{3} \mathrm{BiL}_{2}\right]$ were synthesised by the method reported especially using oxidative addition and complexation reactions.

\section{RESULTS AND DISCUSSION}

The synthesis of tris(pentafluorophenyl)bismuth(III)dicarboxylates was performed in laboratory with the help of the following reactions:

\author{
$\mathrm{R}_{3} \mathrm{Bi}+\mathrm{Cl}_{2} \underset{\mathrm{Et}_{3} \mathrm{~N}}{\stackrel{\text { Direct Chlorination }}{\longrightarrow}} \mathrm{R}_{3} \mathrm{BiCl}$ \\ $\mathrm{R}_{3} \mathrm{BiCl}_{2}+2 \mathrm{HL} \rightarrow \mathrm{R}_{3} \mathrm{BiL}_{2}+2 \mathrm{Et}_{3} \mathrm{~N} \cdot \mathrm{HCl}$ \\ Here: \\ $\mathrm{R}=\left(\mathrm{C}_{6} \mathrm{~F}_{5}\right) ; \mathrm{HL}=($ Respective carboxylic acids $)$.
}

All the newly synthesized tris(pentafluorophenyl)bismuth(V) dicarboxylates were crystalline solids, air stable, and soluble in common organic solvents. The compounds were further characterized by their melting points and analytical techniques such as elemental analysis, infrared, and NMR spectroscopy to ascertain their structures and explore their biological properties. The new compounds have sharp melting points and possess trigonal bipyramidal structure as per results obtained by further analysis.

\section{IR and NMR spectral analysis}

The IR spectra of new tris(pentafluorophenyl)bismuth(V)dicarboxylates were recorded in PerkinElmer spectrophotometer in 4000-200/cm range. The IR spectra of these compounds show absorption bands due to pentafluorophenyl groups. The absorption frequencies have been fully assigned. The Bi-C vibration in case of pentafluorophenyl derivatives corresponding to the "y" mode appears in the range of $440-460 / \mathrm{cm}$. The IR data suggested a monodentate coordination mode of the carboxylate ligands. The ${ }^{1} \mathrm{H}-\mathrm{NMR}$ spectra of the representative tris(pentafluorophenyl)bismuth(V)dicarboxylates showed a multiplet in the range of $\delta 7.82-8.12 \mathrm{ppm}$ which could be assigned to aromatic protons. The ${ }^{19} \mathrm{~F}$-NMR spectra of the compound were carried out at room temperature and the compounds showed peaks appearing in the approximate range consistent with the presence of fluorophenyl groups. Thus, on the basis of above discussions, the newly synthesized compounds assigned a trigonal bipyramidal structure. 
Here,

$\mathrm{R}=\left(\mathrm{C}_{6} \mathrm{~F}_{5}\right) ; \mathrm{L}=$ Respective carboxylate as ligands.

\section{Antibacterial activity}

Antibacterial activity of these compounds was studied against three human pathogenic bacteria, namely, Pseudomonas aeruginosa,

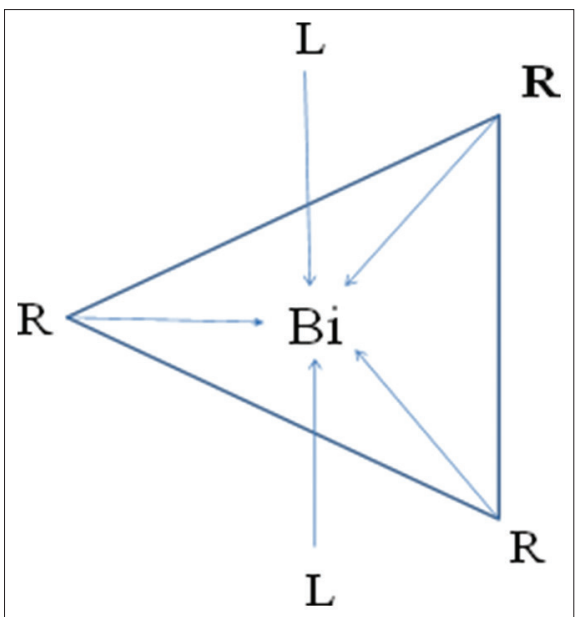

Fig. 1: Suggested structure of triorganobismuth(V) dicarboxylates

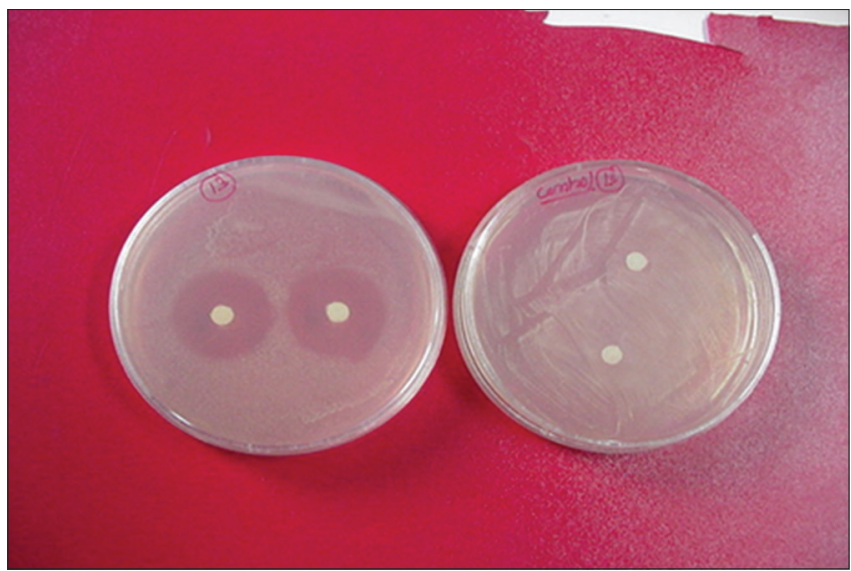

Fig. 2: Antibacterial activity of $\left(\mathrm{C}_{6} \mathrm{~F}_{5}\right)_{3} \mathrm{Bi}\left(\mathrm{OOCC}_{6} \mathrm{H}_{4} \mathrm{~N}\left(\mathrm{C}_{2} \mathrm{H}_{5}\right)_{2}\right)_{2}$

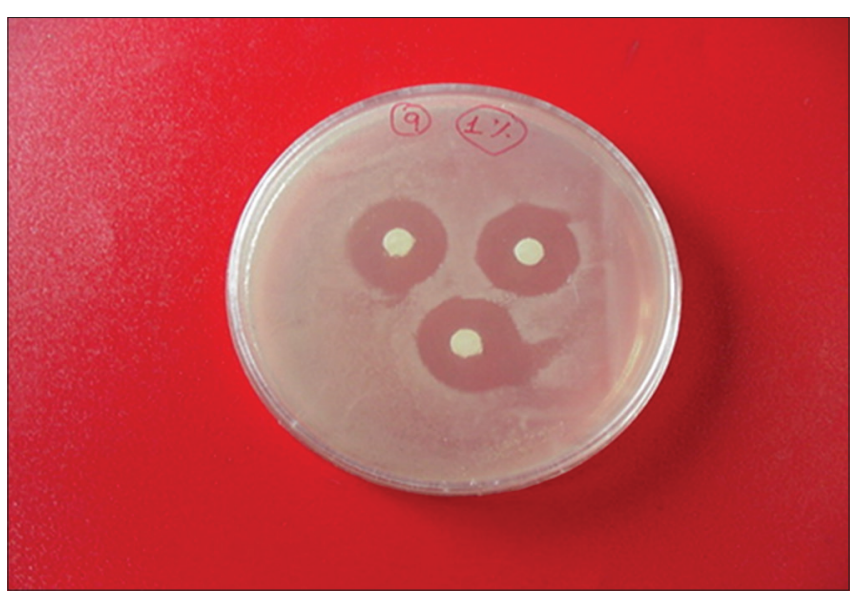

Fig. 3: Antifungal activity of $\left[\left(\mathrm{C}_{6} \mathrm{~F}_{5}\right)_{3} \mathrm{Bi}\left(\mathrm{OOCC}_{6} \mathrm{H}_{3}(\mathrm{OH}) \mathrm{OCH}_{3}\right]_{2}\right.$
Staphylococcus aureus, and Klebsiella pneumonia, using $10 \mathrm{mg} / \mathrm{ml}$ conc. of the test compounds. It was found that compounds show moderate to higher activity against $P$. aeruginosa, $S$. aureus, and $K$. pneumonia. It was found that the respective compounds may damage the cell wall of bacteria by reacting with peptides of cell wall of bacteria.

\section{Antifungal activity}

Antifungal activity of these compounds was tested against two fungal strains, namely, Aspergillus flavus and Aspergillus niger at different concentrations, namely, $10 \mathrm{mg} / \mathrm{ml}, 20 \mathrm{mg} / \mathrm{ml}, 50 \mathrm{mg} / \mathrm{ml}$, and $100 \mathrm{mg} / \mathrm{ml}$ of the test compounds. At $10 \mathrm{mg} / \mathrm{ml}$ conc., the compounds show better inhibition (\%) against A. flavus and A. niger. At $20 \mathrm{mg} / \mathrm{ml}$ concentration of test compounds, the compounds show higher percentage inhibition while at $50 \mathrm{mg} / \mathrm{ml}$ and $100 \mathrm{mg} / \mathrm{ml}$ concentration, approximately all the compounds show higher percentage of inhibition against fungal strains.

\section{In vitro antitumor activity}

The compounds show moderate to high activity against tumor cell lines. It was found that these compounds are in +3 oxidation state and the slight variation in their activity is due to the presence of different carboxylate group as ligand. The compounds generally interact with the receptor site of multienzyme complex responsible for the cytostatic and cytotoxic conditions. It was reported that compounds in +3 oxidation state can easily bind with the receptor site. It may be noted that the organobismuth compound generally binds with nitrogen 7 position of purine bases in DNA molecule and forms complexes with DNA strands affecting replication and transcription of DNA molecule and stops the cell division along with protein synthesis.

\section{Experimental}

The fluorine-based triorganobismuth(V)dichloride was synthesized by the methods reported earlier [16]. The ligands were recrystallized before use while the reactions were performed under inert/nitrogen atmosphere. Preparation of representative organobismuth compounds is discussed below.

\section{Reaction of $\left(\mathrm{C}_{6} \mathrm{~F}_{5}\right)_{3} \mathrm{BiCl}_{2}$ with (HOOC. $\left.\mathrm{C}_{6} \mathrm{H}_{4} \cdot \mathrm{NO}_{2}\right)$}

In an oxygen-free nitrogen atmosphere, solution of tris(pentafluorophenyl)bismuth(V) dichloride $(1 \mathrm{mmol})$ in benzene and 2-nitrobenzoic acid ( $2 \mathrm{mmol}$ ) in same solvent was stirred together in the presence of triethylamine at room temperature for $6 \mathrm{~h}$. The offwhite color $\mathrm{Et}_{3} \mathrm{~N} . \mathrm{HCl}$ was formed (M.P. $=240^{\circ} \mathrm{C}$ ), which was filtered off and the filtrate on evaporation in vacuum gives an off-white color crystalline solid which was further recrystallized in petroleum ether.

\section{Reaction of $\left(\mathrm{C}_{6} \mathrm{~F}_{5}\right)_{3} \mathrm{BiCl}_{2}$ with (HOOC.C $\mathrm{C}_{6} \mathrm{H}_{4} \cdot \mathrm{NO}_{2}$ )}

In an inert atmosphere, solution of tris(pentafluorophenyl)bismuth(V) dichloride $(1 \mathrm{mmol})$ in benzene and 4-nitrobenzoic acid $(2 \mathrm{mmol})$ in same solvent was stirred together in the presence of triethylamine at room temperature for $6 \mathrm{~h}$. The off-white color $\mathrm{Et}_{3} \mathrm{~N} . \mathrm{HCl}$ was formed (M.P. $=240^{\circ} \mathrm{C}$ ), which was filtered off and the filtrate on evaporation in vacuum gives an off-white color crystalline solid which was further recrystallized in petroleum ether.

\section{Reaction of $\left(\mathrm{C}_{6} \mathrm{~F}_{5}\right)_{3} \mathrm{BiCl}_{2}$ with (HOOC.C $\mathrm{C}_{6} \mathrm{H}_{4} \cdot \mathrm{Cl}$ )}

In an oxygen-free nitrogen atmosphere, solution of tris(pentafluorophenyl)bismuth(V) dichloride $(1 \mathrm{mmol})$ in benzene and 2-chlorobenzoic acid ( $2 \mathrm{mmol}$ ) in same solvent was stirred together in the presence of triethylamine at room temperature for $6 \mathrm{~h}$. The offwhite color $\mathrm{Et}_{3} \mathrm{~N} . \mathrm{HCl}$ was formed (M.P. $=240^{\circ} \mathrm{C}$ ), which was filtered off and the filtrate on evaporation in vacuum gives an off-white color crystalline solid which was further recrystallized in petroleum ether.

\section{Reaction of $\left(\mathrm{C}_{6} \mathrm{~F}_{5}\right)_{3} \mathrm{BiCl}_{2}$ with (HOOC. $\left.\mathrm{C}_{6} \mathrm{H}_{4} \cdot \mathrm{Cl}\right)$}

In an oxygen-free nitrogen atmosphere, solution of tris(pentafluorophenyl)bismuth $(\mathrm{V})$ dichloride $(1 \mathrm{mmol})$ in benzene and 4-chlorobenzoic acid (2 mmol) in same solvent was stirred together in the presence of triethylamine at room temperature for 
$6 \mathrm{~h}$. The off-white color $\mathrm{Et}_{3} \mathrm{~N} . \mathrm{HCl}$ was formed (M.P. $=240^{\circ} \mathrm{C}$ ), which was filtered off and the filtrate on evaporation in vacuum gives an off-white color crystalline solid which was further recrystallized in petroleum ether.

\section{Reaction of $\left(\mathrm{C}_{6} \mathrm{~F}_{5}\right)_{3} \mathrm{BiCl}_{2}$ with [(HOOC. $\left.\mathrm{C}_{6} \mathrm{H}_{3}(\mathrm{OH})\left(\mathrm{OCH}_{3}\right)\right]$}

In an inert atmosphere, solution of tris(pentafluorophenyl)bismuth(V) dichloride (1 mmol) in benzene and 3-methxy 4-hydroxybenzoic acid ( $2 \mathrm{mmol}$ ) in same solvent was stirred together in the presence of triethylamine at room temperature for $7 \mathrm{~h}$. The off-white color $\mathrm{Et}_{3} \mathrm{~N}$.
$\mathrm{HCl}$ was formed (M.P. $=240^{\circ} \mathrm{C}$ ), which was filtered off and the filtrate on evaporation in vacuum gives an off-white color crystalline solid which was further recrystallized in petroleum ether.

\section{Reaction of $\left(\mathrm{C}_{6} \mathrm{~F}_{5}\right)_{3} \mathrm{BiCl}_{2}$ with $\left(\mathrm{HOOC} \cdot \mathrm{C}_{6} \mathrm{H}_{4} \cdot \mathrm{NH}_{2}\right)$}

In an oxygen-free nitrogen atmosphere, solution of tris(pentafluorophenyl)bismuth(V) dichloride $(1 \mathrm{mmol})$ in benzene and 2-aminobenzoic acid ( $2 \mathrm{mmol}$ ) in same solvent was stirred together in the presence of triethylamine at room temperature for $6 \mathrm{~h}$. The offwhite color $\mathrm{Et}_{3} \mathrm{~N} . \mathrm{HCl}$ was formed (M.P. $=240^{\circ} \mathrm{C}$ ), which was filtered

Table 1: Physicochemical analysis of triorganobismuth (V) dicarboxylates

\begin{tabular}{|c|c|c|c|c|c|c|}
\hline \multirow[t]{2}{*}{ S. No. } & \multirow[t]{2}{*}{ Compounds } & \multirow{2}{*}{$\begin{array}{l}\text { Yield } \\
\%\end{array}$} & \multirow{2}{*}{$\begin{array}{l}\text { M.P } \\
{ }^{\circ} \mathrm{C}\end{array}$} & \multirow[t]{2}{*}{ Solvent } & \multicolumn{2}{|l|}{$\operatorname{IR}\left(\mathrm{cm}^{-1}\right)$} \\
\hline & & & & & $v_{\text {asym }}(\mathbf{C O})$ & $v_{\text {sym }}(\mathbf{C O})$ \\
\hline 1. & $\left(\mathrm{C}_{6} \mathrm{~F}_{5}\right)_{3} \mathrm{Bi}\left(\mathrm{OOCC}_{6} \mathrm{H}_{4} \mathrm{NO}_{2}\right)_{2}$ & 68 & 62 & Petroleum ether & 1724 vs & $1326 \mathrm{~ms}$ \\
\hline 3. & $\left(\mathrm{C}_{6}^{6} \mathrm{~F}_{5}^{5}\right)_{3} \mathrm{Bi}\left(\mathrm{OOCC}_{6}^{6} \mathrm{H}_{4}^{4} \mathrm{Cl}\right)_{2}^{2}$ & 70 & 63 & Petroleum ether & $1732 \mathrm{~ms}$ & $1330 \mathrm{~ms}$ \\
\hline 4. & $\left(\mathrm{C}_{6}^{6} \mathrm{~F}_{5}^{5}\right)_{3}^{3} \mathrm{Bi}\left(\mathrm{OOCC}_{6}^{6} \mathrm{H}_{4}^{4} \mathrm{Cl}\right)_{2}^{2}$ & 72 & 61 & Petroleum ether & 1709 vs & $1306 \mathrm{~ms}$ \\
\hline 5. & {$\left[\left(\mathrm{C}_{6} \mathrm{~F}_{5}\right)_{3}^{3} \mathrm{Bi}\left(\mathrm{OOCC}_{6} \mathrm{H}_{3}^{4}(\mathrm{OH}) \mathrm{OCH}_{3}\right]_{2}\right.$} & 66 & 64 & Petroleum ether & 1758 vs & $1356 \mathrm{~ms}$ \\
\hline 6. & $\left(\mathrm{C}_{6} \mathrm{~F}_{5}\right)_{3} \mathrm{Bi}\left(\mathrm{OOCC}_{6} \mathrm{H}_{4} \mathrm{NH}_{2}\right)_{2}$ & 65 & 66 & Petroleum ether & $1726 \mathrm{~ms}$ & $1325 \mathrm{~ms}$ \\
\hline 7. & $\left(\mathrm{C}_{6}^{6} \mathrm{~F}_{5}^{5}\right)_{3}^{3} \mathrm{Bi}\left(\mathrm{OOCC}_{6}^{6} \mathrm{H}_{4}^{4} \mathrm{NH}_{2}\right)_{2}$ & 65 & 67 & Petroleum ether & 1729 vs & $1327 \mathrm{~ms}$ \\
\hline 8. & $\left(\mathrm{C}_{6} \mathrm{~F}_{5}\right)_{3} \mathrm{Bi}\left(\mathrm{OOCC}_{6} \mathrm{H}_{4}^{4} \mathrm{~N}\left(\mathrm{CH}_{3}\right)_{2}\right)_{2}$ & 62 & 60 & Petroleum ether & 1752 vs & $1350 \mathrm{~ms}$ \\
\hline 9. & $\left(\mathrm{C}_{6} \mathrm{~F}_{5}\right)_{3} \mathrm{Bi}\left(\mathrm{OOCC}_{6} \mathrm{H}_{4}^{4} \mathrm{~N}\left(\mathrm{C}_{2} \mathrm{H}_{5}\right)_{2}\right)_{2}$ & 60 & 58 & Petroleum ether & $1727 \mathrm{~ms}$ & $1325 \mathrm{~ms}$ \\
\hline
\end{tabular}

Table 2: Antibacterial activity

\begin{tabular}{|c|c|c|c|c|c|}
\hline S. No. & Compounds & Control & Pseudomonas aeruginosa & Staphylococcus aureus & Klebsiella pneumonia \\
\hline 1. & $\left(\mathrm{C}_{6} \mathrm{~F}_{5}\right)_{3} \mathrm{Bi}\left(\mathrm{OOCC}_{6} \mathrm{H}_{4} \mathrm{NO}_{2}\right)_{2}$ & - & ++ & + & ++ \\
\hline 2. & $\left(\mathrm{C}_{6} \mathrm{~F}_{5}\right)_{3} \mathrm{Bi}\left(\mathrm{OOCC}_{6} \mathrm{H}_{4} \mathrm{NO}_{2}\right)_{2}$ & - & ++ & ++ & + \\
\hline 3. & $\left(\mathrm{C}_{6} \mathrm{~F}_{5}\right)_{3} \mathrm{Bi}\left(\mathrm{OOCC}_{6} \mathrm{H}_{4} \mathrm{Cl}\right)_{2}$ & - & + & +++ & ++ \\
\hline 4. & $\left(\mathrm{C}_{6} \mathrm{~F}_{5}\right)_{3} \mathrm{Bi}\left(\mathrm{OOCC}_{6} \mathrm{H}_{4} \mathrm{Cl}\right)_{2}$ & - & ++ & ++ & +++ \\
\hline 5. & {$\left[\left(\mathrm{C}_{6} \mathrm{~F}_{5}\right)_{3}^{3} \mathrm{Bi}\left(\mathrm{OOCC}_{6} \mathrm{H}_{3}(\mathrm{OH}) \mathrm{OCH}_{3}\right]_{2}\right.$} & - & +++ & + & ++ \\
\hline 6. & $\left(\mathrm{C}_{6} \mathrm{~F}_{5}\right)_{3} \mathrm{Bi}\left(\mathrm{OOCC}_{6} \mathrm{H}_{4} \mathrm{NH}_{2}\right)_{2}$ & - & ++ & ++ & ++ \\
\hline 7. & $\left(\mathrm{C}_{6} \mathrm{~F}_{5}\right)_{3} \mathrm{Bi}\left(\mathrm{OOCC}_{6} \mathrm{H}_{4} \mathrm{NH}_{2}\right)_{2}$ & - & ++ & ++ & +++ \\
\hline 8. & $\left(\mathrm{C}_{6} \mathrm{~F}_{5}\right)_{3} \mathrm{Bi}\left(\mathrm{OOCC}_{6} \mathrm{H}_{4} \mathrm{~N}\left(\mathrm{CH}_{3}\right)_{2}\right)_{2}$ & - & ++ & ++ & +++ \\
\hline 9. & $\left(\mathrm{C}_{6} \mathrm{~F}_{5}\right)_{3} \mathrm{Bi}\left(\mathrm{OOCC}_{6} \mathrm{H}_{4} \mathrm{~N}\left(\mathrm{C}_{2} \mathrm{H}_{5}\right)_{2}\right)_{2}$ & - & +++ & +++ & +++ \\
\hline
\end{tabular}

+: 6-10 mm (dia), ++: 10-14 mm, +++: >14 mm, “-": Inactive; (control)

Table 3: Antifungal activity of $10 \mu \mathrm{g} / \mathrm{ml}$ conc. of compound

\begin{tabular}{|c|c|c|c|c|c|}
\hline S. No. & Compounds & $\begin{array}{l}\text { Aspergillus flavus } \\
\text { col. dia. (mm) }\end{array}$ & $\%$ inhibition & $\begin{array}{l}\text { Aspergillus niger } \\
\text { col. dia. (mm) }\end{array}$ & $\%$ inhibition \\
\hline 1. & $\left(\mathrm{C}_{6} \mathrm{~F}_{5}\right)_{3} \mathrm{Bi}\left(\mathrm{OOCC}_{6} \mathrm{H}_{4} \mathrm{NO}_{2}\right)_{2}$ & 1.2 & 60.0 & 1.0 & 50.0 \\
\hline 2. & $\left(\mathrm{C}_{6} \mathrm{~F}_{5}\right)_{3} \mathrm{Bi}\left(\mathrm{OOCC}_{6} \mathrm{H}_{4}^{4} \mathrm{NO}_{2}\right)_{2}$ & 1.4 & 53.3 & 1.5 & 25.0 \\
\hline 3. & $\left(\mathrm{C}_{6}^{0} \mathrm{~F}_{5}\right)_{3} \mathrm{Bi}\left(\mathrm{OOCC}_{6} \mathrm{H}_{4}^{4} \mathrm{Cl}\right)_{2}$ & 1.4 & 53.3 & 1.0 & 50.0 \\
\hline 4. & $\left(\mathrm{C}_{6} \mathrm{~F}_{5}\right)_{3} \mathrm{Bi}\left(\mathrm{OOCC}_{6} \mathrm{H}_{4} \mathrm{Cl}\right)_{2}$ & 1.2 & 60.0 & 1.4 & 30.0 \\
\hline 5. & {$\left[\left(\mathrm{C}_{6} \mathrm{~F}_{5}\right)_{3}{ }_{3} \mathrm{Bi}\left(\mathrm{OOCC}_{6} \mathrm{H}_{3}(\mathrm{OH}) \mathrm{OCH}_{3}\right]_{2}\right.$} & 1.2 & 60.0 & 1.5 & 25.0 \\
\hline 6. & $\left(\mathrm{C}_{6} \mathrm{~F}_{5}\right)_{3} \mathrm{Bi}\left(\mathrm{OOCC}_{6} \mathrm{H}_{4} \mathrm{NH}_{2}\right)_{2}$ & 0.8 & 73.3 & 1.4 & 30.0 \\
\hline 8. & $\left(\mathrm{C}_{6} \mathrm{~F}_{5}\right)_{3} \mathrm{Bi}\left(\mathrm{OOCC}_{6} \mathrm{H}_{4}^{4} \mathrm{~N}\left(\mathrm{CH}_{3}\right)_{2}\right)_{2}$ & 1.2 & 60.0 & 1.0 & 50.0 \\
\hline 9. & $\left(\mathrm{C}_{6} \mathrm{~F}_{5}\right)_{3} \mathrm{Bi}\left(\mathrm{OOCC}_{6} \mathrm{H}_{4} \mathrm{~N}\left(\mathrm{C}_{2} \mathrm{H}_{5}\right)_{2}\right)_{2}$ & 0.8 & 73.3 & 1.2 & 40.0 \\
\hline 10. & Control & 3.0 & - & 2.0 & - \\
\hline
\end{tabular}

Table 4: Antifungal activity of $20 \mu \mathrm{g} / \mathrm{ml}$ conc. of compound

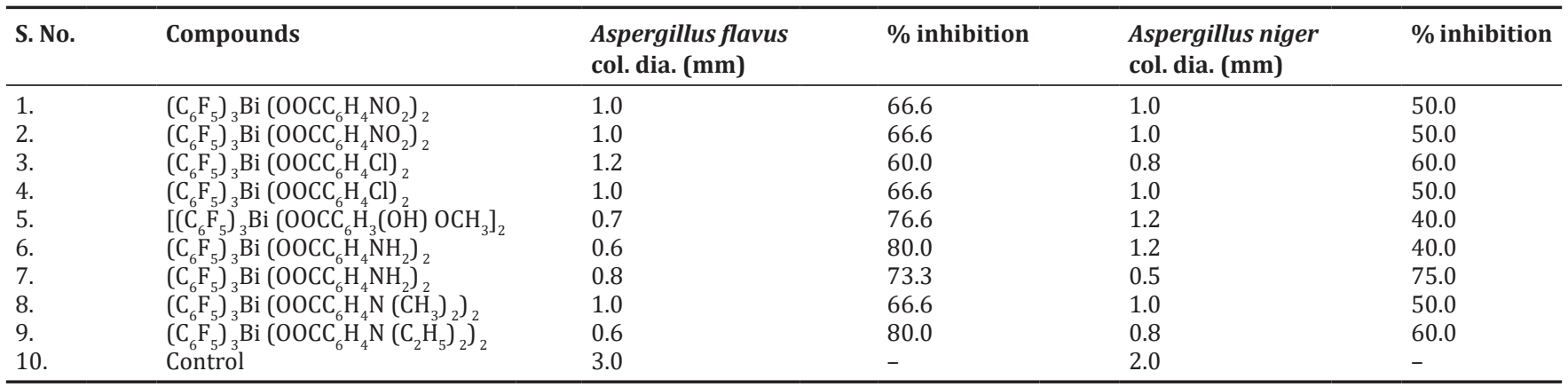


Table 5: Antifungal activity of $50 \mu \mathrm{g} / \mathrm{ml}$ conc. of compound

\begin{tabular}{|c|c|c|c|c|c|}
\hline S. No. & Compounds & $\begin{array}{l}\text { Aspergillus flavus } \\
\text { col. dia. }(\mathrm{mm})\end{array}$ & $\%$ inhibition & $\begin{array}{l}\text { Aspergillus niger } \\
\text { col. dia. }(\mathrm{mm})\end{array}$ & $\%$ inhibition \\
\hline 1. & $\left(\mathrm{C}_{6} \mathrm{~F}_{5}\right)_{3} \mathrm{Bi}\left(\mathrm{OOCC}_{6} \mathrm{H}_{4} \mathrm{NO}_{2}\right)_{2}$ & 0.6 & 80.0 & 0. & 75.0 \\
\hline 2. & $\left(\mathrm{C}_{6} \mathrm{~F}_{5}\right)_{3} \mathrm{Bi}\left(\mathrm{OOCC}_{6} \mathrm{H}_{4} \mathrm{NO}_{2}\right)_{2}$ & 0.7 & 76.6 & 0.6 & 70.0 \\
\hline 3. & $\left(\mathrm{C}_{6}^{6} \mathrm{~F}_{5}\right)_{3} \mathrm{Bi}\left(\mathrm{OOCC}_{6}^{6} \mathrm{H}_{4}^{4} \mathrm{Cl}\right)_{2}^{2}$ & 1.0 & 66.6 & 0.5 & 75.0 \\
\hline 4. & $\left(\mathrm{C}_{6} \mathrm{~F}_{5}\right)_{3} \mathrm{Bi}\left(\mathrm{OOCC}_{6} \mathrm{H}_{4} \mathrm{Cl}\right)_{2}$ & 0.8 & 73.3 & 0.8 & 60.0 \\
\hline 5. & {$\left[\left(\mathrm{C}_{6} \mathrm{~F}_{5}\right)_{3} \mathrm{Bi}\left(\mathrm{OOCC}_{6} \mathrm{H}_{3}(\mathrm{OH}) \mathrm{OCH}_{3}\right]_{2}\right.$} & 0.5 & 83.3 & 0.8 & 60.0 \\
\hline 6. & $\left(\mathrm{C}_{6} \mathrm{~F}_{5}\right)_{3} \mathrm{Bi}\left(\mathrm{OOCC}_{6} \mathrm{H}_{4} \mathrm{NH}_{2}\right)_{2}$ & 0.4 & 86.7 & 0.5 & 75.0 \\
\hline 8. & $\left(\mathrm{C}_{6} \mathrm{~F}_{5}\right)_{3} \mathrm{Bi}\left(\mathrm{OOCC}_{6} \mathrm{H}_{4}^{4} \mathrm{~N}\left(\mathrm{CH}_{3}\right)_{2}\right)_{2}$ & 0.5 & 83.3 & 0.8 & 60.0 \\
\hline 9. & $\left(\mathrm{C}_{6} \mathrm{~F}_{5}\right)_{3} \mathrm{Bi}\left(\mathrm{OOCC}_{6} \mathrm{H}_{4}^{4} \mathrm{~N}\left(\mathrm{C}_{2} \mathrm{H}_{5}\right)_{2}\right)_{2}$ & 0.4 & 86.7 & 0.6 & 70.0 \\
\hline 10. & Control & 3.0 & - & 2.0 & - \\
\hline
\end{tabular}

Table 6: Antifungal activity of $100 \mu \mathrm{g} / \mathrm{ml}$ conc. of compounds

\begin{tabular}{|c|c|c|c|c|c|}
\hline S. No. & Compounds & $\begin{array}{l}\text { Aspergillus flavus } \\
\text { col. dia. (mm) }\end{array}$ & $\%$ inhibition & $\begin{array}{l}\text { Aspergillus niger } \\
\text { col. dia. (mm) }\end{array}$ & $\%$ inhibition \\
\hline 1. & $\left(\mathrm{C}_{6} \mathrm{~F}_{5}\right)_{3} \mathrm{Bi}\left(\mathrm{OOCC}_{6} \mathrm{H}_{4} \mathrm{NO}_{2}\right)_{2}$ & 0.4 & 86.7 & 0.2 & 90.0 \\
\hline 2. & $\left(\mathrm{C}_{6} \mathrm{~F}_{5}\right)_{3} \mathrm{Bi}\left(\mathrm{OOCC}_{6} \mathrm{H}_{4} \mathrm{NO}_{2}\right)_{2}$ & 0.4 & 86.7 & 0.1 & 95.0 \\
\hline 3. & $\left(\mathrm{C}_{6}^{6} \mathrm{~F}_{5}^{5}\right)_{3} \mathrm{Bi}\left(\mathrm{OOCC}_{6}^{6} \mathrm{H}_{4}^{4} \mathrm{Cl}\right)_{2}^{2}$ & 0.8 & 73.3 & 0.2 & 90.0 \\
\hline 4. & $\left(\mathrm{C}_{6} \mathrm{~F}_{5}\right)_{3} \mathrm{Bi}\left(\mathrm{OOCC}_{6} \mathrm{H}_{4} \mathrm{Cl}\right)_{2}$ & 0.5 & 83.3 & 0.4 & 80.0 \\
\hline 5. & {$\left[\left(\mathrm{C}_{6} \mathrm{~F}_{5}\right)_{3}^{3} \mathrm{Bi}\left(\mathrm{OOCC}_{6} \mathrm{H}_{3}(\mathrm{OH}) \mathrm{OCH}_{3}\right]_{2}\right.$} & 0.1 & 96.7 & 0.5 & 75.0 \\
\hline 6. & $\left(\mathrm{C}_{6} \mathrm{~F}_{5}\right)_{3} \mathrm{Bi}\left(\mathrm{OOCC}_{6} \mathrm{H}_{4} \mathrm{NH}_{2}\right)_{2}$ & 0.2 & 93.3 & 0.2 & 90.0 \\
\hline 7. & $\left(\mathrm{C}_{6}^{6} \mathrm{~F}_{5}\right)_{3} \mathrm{Bi}\left(\mathrm{OOCC}_{6}^{6} \mathrm{H}_{4}^{4} \mathrm{NH}_{2}\right)_{2}$ & 0.1 & 96.7 & 0.1 & 95.0 \\
\hline 8. & $\left(\mathrm{C}_{6} \mathrm{~F}_{5}\right)_{3} \mathrm{Bi}\left(\mathrm{OOCC}_{6} \mathrm{H}_{4} \mathrm{~N}\left(\mathrm{CH}_{3}\right)_{2}\right)_{2}$ & 0.4 & 86.7 & 0.5 & 75.0 \\
\hline 9. & $\left(\mathrm{C}_{6}^{6} \mathrm{~F}_{5}\right)_{3} \mathrm{Bi}\left(\mathrm{OOCC}_{6} \mathrm{H}_{4} \mathrm{~N}^{4}\left(\mathrm{C}_{2} \mathrm{H}_{5}\right)_{2}\right)_{2}$ & 0.2 & 93.3 & 0.4 & 80.0 \\
\hline 10. & Control & 3.0 & - & 2.0 & - \\
\hline
\end{tabular}

Table 7: Antitumor activity

\begin{tabular}{|c|c|c|c|c|}
\hline S. No. & Compounds & MCF-7 (cell no. $\left.\times 10^{4}\right)$ & EVSA-7 (cell no. $\times 10^{4}$ ) & Activity \\
\hline 1. & $\left(\mathrm{C}_{6} \mathrm{~F}_{5}\right)_{2} \mathrm{Bi}\left(\mathrm{OOCC}_{6} \mathrm{H}_{4} \mathrm{NO}_{2}\right)$ & $8.79 \pm 0.52$ & $8.42 \pm 0.46$ & Positive \\
\hline 2. & $\left(\mathrm{C}_{6} \mathrm{~F}_{5}\right)_{2} \mathrm{Bi}\left(\mathrm{OOCC}_{6} \mathrm{H}_{4} \mathrm{NO}_{2}\right)$ & $9.19 \pm 0.92$ & $9.29 \pm 0.88$ & Positive \\
\hline 3. & $\left(\mathrm{C}_{6} \mathrm{~F}_{5}\right)_{2} \mathrm{Bi}\left(\mathrm{OOCC}_{6} \mathrm{H}_{4} \mathrm{Cl}\right)$ & $8.95 \pm 0.67$ & $8.55 \pm 0.62$ & Positive \\
\hline 4. & $\left(\mathrm{C}_{6} \mathrm{~F}_{5}\right)_{2} \mathrm{Bi}\left(\mathrm{OOCC}_{6} \mathrm{H}_{4}^{4} \mathrm{Cl}\right)$ & $12.31 \pm 1.02$ & $12.39 \pm 1.03$ & Negative \\
\hline 5. & {$\left[\left(\mathrm{C}_{6} \mathrm{~F}_{5}\right)_{2} \mathrm{Bi}\left(\mathrm{OOCC}_{6} \mathrm{H}_{3}(\mathrm{OH}) \mathrm{OCH}_{3}\right]\right.$} & $8.79 \pm 0.52$ & $8.42 \pm 0.46$ & Positive \\
\hline 6. & $\left(\mathrm{C}_{6} \mathrm{~F}_{5}\right)_{2} \mathrm{Bi}\left(\mathrm{OOCC}_{6} \mathrm{H}_{4} \mathrm{NH}_{2}\right)$ & $9.29 \pm 0.88$ & $9.89 \pm 0.92$ & Positive \\
\hline 7. & $\left(\mathrm{C}_{6}^{6} \mathrm{~F}_{5}\right)_{2} \mathrm{Bi}\left(\mathrm{OOCC}_{6}^{6} \mathrm{H}_{4}^{4} \mathrm{NH}_{2}\right)$ & $8.95 \pm 0.67$ & $8.55 \pm 0.62$ & Positive \\
\hline 9. & $\left(\mathrm{C}_{6} \mathrm{~F}_{5}\right)_{2} \mathrm{Bi}\left(\mathrm{OOCC}_{6} \mathrm{H}_{4} \mathrm{~N}\left(\mathrm{C}_{2} \mathrm{H}_{5}\right)_{2}\right)$ & $9.19 \pm 0.92$ & $9.29 \pm 0.88$ & Positive \\
\hline 10. & Negative control & $10.21 \pm 1.01$ & $10.22 \pm 1.01$ & - \\
\hline 11. & Positive control & $40.26 \pm 3.23$ & $41.23 \pm 3.28$ & - \\
\hline
\end{tabular}

*Negative control - Culture medium only, **Positive control - $17 \beta$ estradiol

off and the filtrate on evaporation in vacuum gives an off-white color crystalline solid which was further recrystallized in petroleum ether

\footnotetext{
Reaction of $\left(\mathrm{C}_{6} \mathrm{~F}_{5}\right)_{3} \mathrm{BiCl}_{2}$ with (HOOC. $\left.\mathrm{C}_{6} \mathrm{H}_{4} \cdot \mathrm{NH}_{2}\right)$

In an oxygen-free nitrogen atmosphere, solution of tris(pentafluorophenyl)bismuth(V) dichloride $(1 \mathrm{mmol})$ in benzene and 4-aminobenzoic acid (1 $\mathrm{mmol}$ ) in same solvent was stirred together in the presence of triethylamine at room temperature for $6 \mathrm{~h}$. The offwhite color $\mathrm{Et}_{3} \mathrm{~N} . \mathrm{HCl}$ was formed (M.P. $=240^{\circ} \mathrm{C}$ ), which was filtered off and the filtrate on evaporation in vacuum gives an off-white color crystalline solid which was further recrystallized in petroleum ether.
}

\section{Reaction of $\left(\mathrm{C}_{6} \mathrm{~F}_{5}\right)_{3} \mathrm{BiCl}_{2}$ with [(HOOC. $\left.\left.\mathrm{C}_{6} \mathrm{H}_{4} \cdot \mathrm{N}\left(\mathrm{CH}_{3}\right)_{2}\right)\right]$}

In an oxygen-free nitrogen atmosphere, solution of tris(pentafluorophenyl)bismuth(V) dichloride $(1 \mathrm{mmol})$ in benzene and 3-dimethylaminobenzoic acid ( $2 \mathrm{mmol}$ ) in same solvent was stirred together in the presence of triethylamine at room temperature for $7 \mathrm{~h}$. The off-white color $\mathrm{Et}_{3} \mathrm{~N} . \mathrm{HCl}$ was formed (M.P. $=240^{\circ} \mathrm{C}$ ), which was filtered off and the filtrate on evaporation in vacuum gives an off-white color crystalline solid which was further recrystallized in petroleum ether.
Reaction of $\left(\mathrm{C}_{6} \mathrm{~F}_{5}\right)_{3} \mathrm{BiCl}_{2}$ with [(HOOC. $\left.\left.\mathrm{C}_{6} \mathrm{H}_{4} \cdot \mathrm{N}\left(\mathrm{C}_{2} \mathrm{H}_{5}\right)_{2}\right)\right]$

In an oxygen-free nitrogen atmosphere, solution of tris(pentafluorophenyl)bismuth(V) dichloride $(1 \mathrm{mmol})$ in benzene and 4-diethylaminobenzoic acid ( $2 \mathrm{mmol})$ in same solvent was stirred together in the presence of triethylamine at room temperature for 4-5 h. The off-white color $\mathrm{Et}_{3} \mathrm{~N} \cdot \mathrm{HCl}$ was formed (M.P. $=240^{\circ} \mathrm{C}$ ), which was filtered off and the filtrate on evaporation in vacuum gives an off-white color crystalline solid which was further recrystallized in petroleum ether.

\section{Antibacterial activity}

Antibacterial activity of these compounds was determined by disk diffusion method [17]. In this technique, the filter paper (Whatman No. 1) sterile discs of $5 \mathrm{~mm}$ diameter, impregnated with the test compounds $\left(10 \mathrm{mg} / \mathrm{ml}\right.$ of ethanol), were placed on the nutrient agar plate at $37^{\circ} \mathrm{C}$ for $24 \mathrm{~h}$. The inhibition zones around the dried impregnated discs were measured after $24 \mathrm{~h}$. The activity was classified as "highly active" (diameter $>14 \mathrm{~mm}$ ); "moderately active" (diameter $=10-14 \mathrm{~mm}$ ), and "slightly active" (diameter $=6-10$ ). The diameter less than $6 \mathrm{~mm}$ was regarded as inactive. 


\section{Antifungal activity}

The antifungal activity of these compounds was tested by agar diffusion method [18] using four concentrations of the tests compounds, namely, $10,20,50$, and $100 \mathrm{mg} / \mathrm{ml}$; against the two human pathogenic fungi, $A$. flavus and A. niger. The $1 \mathrm{ml}$ of each compound was poured into a Petri dish having about $20-25 \mathrm{ml}$ of molten agar medium of potato dextrose. As the medium gets solidify, Petri dishes were inoculated separately with the fungal isolates and kept at $26^{\circ} \mathrm{C}$ for $96 \mathrm{~h}$. All the values (\% inhibition) were recorded. The $\%$ inhibition of these compounds was calculated using the following mathematical equation.

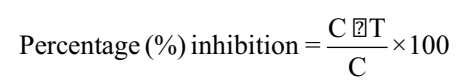

Here,$\quad C=$ Diameter of fungus in control and $\mathrm{T}=$ Diameter of fungus in test compounds.

\section{Antitumor studies}

The in vitro antitumor activity of these compounds was carried out by 3-(4,5-dimethylthiazol-2-yl)-2,5-diphenyl tetrazolium bromide (MTT) method [19]. This method was performed to estimate the effect of compounds on the growth of cell. The human breast adenocarcinoma (MCF-7) and mammary cancer (EVSA-7) cell lines were used for this purpose. The principle behind this assay depends on the reduction of tetrazolium salt. The yellow-colored tetrazolium MTT was reduced partially by metabolically active cells by the action of dehydrogenase enzyme to generate NADH and NADPH as reducing equivalents. The resulting intracellular purple color zone was solubilized and quantified by spectrophotometer. The MTT was first dissolved in phosphate buffer saline at a concentration of $5 \mathrm{mg} / \mathrm{ml}$. The MTT solution $(50 \mathrm{ml})$ was added to each well of 96-well culture plate containing $100 \mathrm{ml}$ of culture medium and incubates at $37^{\circ} \mathrm{C}$ for $4 \mathrm{~h}$. The medium was then removed carefully without disturbing the crystals of purple-colored zone then 50 $\mathrm{ml}$ of DMSO was added to each well and mixed thoroughly to dissolve the crystals of the zone. The plate was then read on a micro-ELISA plate reader at a wavelength of $570 \mathrm{~nm}$ to fine out the optical density and cell count value.

\section{CONCLUSION}

The newly synthesized organobismuth(V) substituted carboxylates show trigonal bipyramidal structure as per elemental and spectral analysis. The compounds were also show potent antimicrobial and antitumor activity.

\section{ACKNOWLEDGMENT}

The authors are highly thankful to the Vice-Chancellor, Shri Ramswaroop Memorial University, for providing necessary laboratory facility and also thankful to the Director, Helix BioGenesis Pvt. Ltd., for antimicrobial and antitumor studies.

\section{AUTHORS' CONTRIBUTIONS}

One of the authors Dipti Mani Tripathi worked as research scholar under the supervision of rest two authors Ravi Kant and Krishna Srivastava to complete this research work.

\section{CONFLICTS OF INTEREST}

There are no conflicts of interest.

\section{AUTHORS' FUNDING}

The research work performed by authors by self-financial scheme and no financial assistance received from any funding agencies.

\section{REFERENCES}

1. Rani P, Gupta $P$, Kant R. Synthesis, characterization and biological screening of some novel complexes of group 15 elements (As, Sb, Bi). Int J Microbiol Res 2017;9:984-6.

2. Rani P, Gupta P, Kant R. Synthesis and characterization of some novel complexes of fluorobenzoic acid with group-15 elements (As, $\mathrm{Sb}, \mathrm{Bi}$ ) for antimicrobial and antitumor studies. Int J Drug Discov 2017;8:250- 3

3. Gupta P, Rani P, Kant R. Antimicrobial studies of some novel organobismuth compounds. Int J Microbiol Res 2018;10:773-5.

4. Gupta P, Rani P, Kant R. Synthesis, characterization and antimicrobial studies of novel organobismuth (III) complexes. Int J Microbiol Res 2018;10:1086-9.

5. Soni KK, Kant R, Kumar A, Synthesis and characterization of novel organobismuth compounds: Antimicrobial and antitumor studies. Int J Chem Res 2015;7:159-63.

6. Soni KK, Kant R, Kumar A. Biomedicinal and gastroprotective studies of some fluorine based diorganobismuth (III) compounds. Int J Chem Res 2016;8:168-72.

7. Tripathi DM, Amresh G, Kant R. Bio-medicinal studies of some new triorganobismuth (V) compounds. Int J Chem Res 2012;4:110-3.

8. Tripathi DM, Amresh G, Kant R. Antimicrobial and antitumor studies of some organic derivatives of bismuth. J Med Chem Lett 2011;1:1- 6 .

9. Tiwari VK, Shukla SK, Chauhan VS, Kant R, Rani S, Tewari IC. Antimicrobial, antitumor and gastro protective studies of some new water soluble organic derivatives of bismuth. Int J Pharma Anal 2010;2:9-14

10. Chauhan VS, Tiwari VK, Shukla SK, Rani S, Mishra SN, Kant R, et al. Biological activity of some organic derivatives of bismuth. Int J Biotechnol Res 2009;2:67-71.

11. Singh S, Hasan SM, Kant R. An overview of chemical properties and pharmacological importance of organobismuth compounds. Chron Pharm Sci 2018;2:35-45.

12. Singh S, Hasan SM, Kant R. Biomedicinal and gastroprotective aspects of organobismuth compounds: Recent approaches and future perspectives. Asian J Pharm Clin Res 2019;12:172-81.

13. Chandrasekhar K, Behl HM, Sidhu OP, Ch VR, Pushpangadan P, Kant R, et al. A Novel Compound as Insecticidal and its Processing. United States: PCT (Global) Patent; 2006.

14. Kant R, Srivastava K, Singh S, Hasan SM, Srivastava J. Organobismuth Compounds for Medical Applications and Preparation Methods Thereof. New Delhi: Indian Patent; 2018.

15. Srivastava K, Kant R. Novel Antitumor Organobismuth Compound and Method of Preparation Therof. New Delhi: Indian Patent; 2019.

16. Singhal K, Kant R, Goswami AK, Raj P. Synthesis and characterization of bis (pentafluorophenyl) antimony (V) cations $\left[\left(\mathrm{C}_{6} \mathrm{~F}_{5}\right)_{2} \mathrm{SbL}_{3}\right]^{3+}$. J Flourine Chem 2003;122:229-32.

17. Kant R, Amresh G, Chandrashekar K, Anil KK. Synthesis and biological activity on some organoantimony (III) compounds. Phosphorus Sulfur Silicon Relat Elem 2008;183:1410-9.

18. Kant R, Amresh G, Chandrashekar K, Shukla SK. Biological studies of some perfluorophenyl antimony compounds. Phosphorus Sulfur Silicon Relat Elem 2009;184:2453-64.

19. Amresh G, Husain Z, Gupta RJ, Kant R, Rao CV, Singh PN. Gastroprotective effects of ethanolic extract from Cissampelos pareira in experimental animals. J Nat Med 2007;61:323-8 